\title{
Téoros
}

Revue de recherche en tourisme

\section{Un mariage de technologies en devenir}

Daniel Y. Zann

Volume 5, numéro 3, novembre 1986

La gestion touristique : les nouvelles technologies

URI : https://id.erudit.org/iderudit/1080534ar

DOI : https://doi.org/10.7202/1080534ar

Aller au sommaire du numéro

Éditeur(s)

Université du Québec à Montréal

ISSN

0712-8657 (imprimé)

1923-2705 (numérique)

Découvrir la revue

Citer cet article

Zann, D. Y. (1986). Un mariage de technologies en devenir. Téoros, 5(3), 14-16. https://doi.org/10.7202/1080534ar d'utilisation que vous pouvez consulter en ligne.

https://apropos.erudit.org/fr/usagers/politique-dutilisation/ 


\title{
Un mariage de technologies en devenir
}

\author{
par Daniel Y. Zann*
}

\section{Tourisme et nouvelles technologie}

Quand une revue spécialisće dans les question de tourisme consacre l'un de ses numéros aux technologies de l'information, il faut naturellement s'interroger sur le rôle des médias électroniques dans la mise en marché des produits touristiques et sur les applications de l'informatique dans les services offerts aux touristes.

En ce qui concerne la promotion touristique, l'influence d'une campagne publicitaire à la télévision n'est plus à démontrer pour rejoindre une vaste clientèle dans les marchés-cibles visés; en outre, on connait mal l'impact que le vidéodisque aura finalement comme outil de vente auprès des grossistes et du grand public. Quant aux services directs aux touristes, on connaît l'efficacité des systemes informatiques de réservations que les grandes compagnies aériennes, les chainnes hôtelières internationales et les entreprises de location d'automobiles ont implantées à travers le monde pour attirer et retenir les clients ă l'interieur de leur réseau.

Une stratégie de mise en marché bien orchestrée retiendra naturellement une combinaison de moyens différents pour s'attaquer à chaque marché. Les outils de promotion les plus fréquemment utilisés aujourd hui comprennent: les annonces ou les publi-reportages à la télévision, dans la presse et dans les revues pour la publicité de masse; la distribution de brochures et prospectus dans les kiosques d'information, les bureaux de tourisme et les hôtels; les missions de prospection et les bourses de voyages pour les intermédiaires, grossistes ou organisateurs de congrès et enfin les centres de renseignements téléphoniques et postaux

Tous ces moyens utilisent les médias conventionnels de la presse électronique, de la presse ecrite et de l'audiovisuel, et jusqu'à maintenant les services télématiques et les

"M. Daniel Zann est actuellement vice-président de la firme Dousery Teleoom; Mme Josette Guimond et M. Pierre Parent du Palais des Congres ont aussi collaborés a la rédaction de cot article.

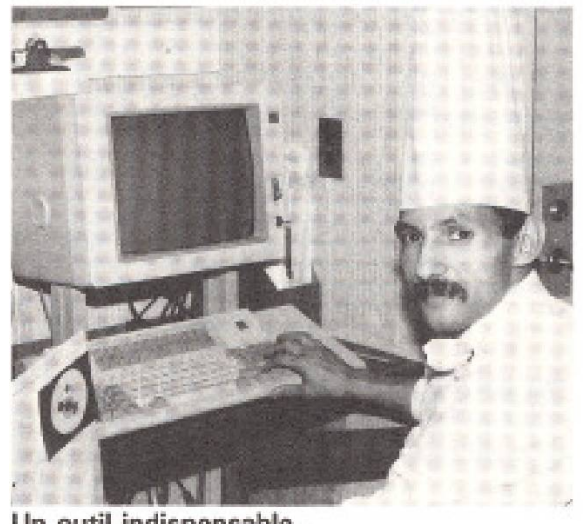

Un outil indispensable

systèmes d'information électroniques ne jouent pas encore, du moins au Canada, le rôle complémentaire auquel leurs possibilités techniques, largement supérieures à celles des autres médias, permettent d'aspirer.

Notre propos ici n'étant pas d'épiloguer sur l'usage que l'industrie du tourisme fait des médias traditionnels mais d'élaborer plutôt sur le potentiel nouveau que la diffusion grandissante des micro-ordinateurs individucls lui apporte grace an mariage de la technologie et des télécommunications avec les techniques de l'informatique; nous consacrerons la suite de nos discussions aux applications de la télénatique dans le secteur du tourisme.

\section{Quelques définitions}

Nous parlons déjà dans ce jargon de spécialistes à la mode. Le profane sait-il au juste quelles réalités d'aujourd'hui ou de demain la télématique, la bureautique, le vidéotex et le vidéodisque recouvrent-ils?

Nous tenterons donc de démystifier au préalable ces termes par quelques courtes deffinitions:

\section{Télématique}

Néologisme désignant l'ensemble des services qui utilisent à la fois les télécommunications (télé) et l'informatique (matique).

\section{Bureautique}

Traduction de l'expression anglo-saxonne "office automation" , la bureautique désigne l'assistance aux travaux de bureau pro- curée par des moyens ou des procédures faisant appel aux techniques de l'électronique, de l'informatique, des télécommunications et de l'organisation administrative. Elle englobe le traitement de texte, le courrier et la messagerie électroniques, l'archivage de documents, la télécopie, etc.

\section{Vidéotex}

Terme dếsignant les systèmes d'information publics ou privés permettant l'affichage sur un écran de télévision de pages d'information organises en magazine dans la mémoire d'un ordinateur qu'on peut interroger à l'aide d'un clavier numérique el d'un décodeur ou d'un micro-ordinateur.

\section{Vidéodisque}

Support de grande capacité permettant l'enregistrement de films ou de diapositi= ves dont l'accès est facilement controblé par un micro-ordinateur.

\section{Les projets liés au tourisme}

Plusieurs systèmes vidéotex à travers le monde offrent des services d'information et de réservations liés au tourisme et aux voyages. Parmi ceux-ci, nous mentionnerons simplement à titre d'exemples les quelques réalisations suivantes.

\section{Au Canada}

Téléguide à Toronto

Date de lancement

Travel Manitoba

Vacation Planner

Info Montréal au

Palais des Congrès

Aux États-Unis

Viewtron en Floride

En Grande-Bretagne

Système Prestel

\section{En France}

Réseau Télétel

Terminal Minitel

Communications Canada fut largement responsable du developpement du système Vidéotex au Canada.

Au Québec, le Palais des Congrès de Mon- 
tréal joue, depuis son ouverture au public en mai 1983, un rôle de pionnier dans l'utilisation de la télématique pour offrir aux délégués, aux exposants et à tous les visiteurs un service d'information et de communication électronique unique au monde.

Cette information électronique s'articule autour de deux applications télématiques principales: la signalisation dynamique et la banque de renseignements touristiques "Info Montréal", dont les possibilités se combinent aux installations audiovisuelles traditionnelles pour permettre la diffusion des messages dans l'enceinte du Palais des Congrès et à des endroits de la ville à forte influence touristique ainsi que la projection sur des écrans de télévision géants, d'images créés par ordinateur.

La signalisation dynamique utilise quelque 72 monitcurs de télévision placés dans des endroits stratégiques du Palais pour guider les visiteurs et les délégués depuis leur entréce jusqu'à leur destination. Ces moniteurs servent également à la diffusion des. changements de derniere minute aux programmes des activités, de bulletins d'information pour les organisateurs et de messages publicitaires pour les exposants et les commanditaires.

La banque de renseignements touristiques Info Montreal contient plus de 4000 pages d'information bilingue constamment mises à jour dans la mémoire de l'ordinateur. Elle contient une foule de renseignements utiles sur les centres d ${ }^{+}$attraction, les activités culturelles, les spectacles. les restaurants, les événements sportifs, etc., qui sont susceptibles d'intéresser les délégués et les visiteurs de passage à Montréal. Toutes ces informations sont accessibles instantanément grấce à 25 consoles d'information d'accès public installees au Palais des Congrès, dans quelques grands hottels du centre-ville et dans certains lieux publics à Montréal. CIDEM-Tourisme fait la cueillette d'informations et le Palais en assure le traitement graphique et la diffusion.

\section{Efforts gouvernementaux}

Par ailleur's, le Ministère du Tourisme du Québec entreprenait à l'automne 1984 une série d'études de faisabilité pour le développement au Québec d'un système de réservations d'hébergement et d'information touristique qui viendrait supporter le plan de mise en marché des produits touristiques du Québec, que la Direction générale du Tourisme a préparé en collaboration avec ses principaux partenaires de 1 "industrie.

On attend maintenant une décision du Gouvernement du Québec quant à la poursuite du projet avec les solutions technologiques les plus prometteuses, et à l'organisation administrative à mettre en place pour en assurer le succès, non seulement sur le plan technique mais aussi et surtout sur le plan de ses retombées économiques sur l'industrie touristique québécoise.

Sans présumer des décisions qui seront prises, il est toutefois vraisemblable de croire que, si ce projet se matérialise, les solutions qui pourraient être retenues s'articulerons autour des possibilités offertes par la microinformatique, le vidéodisque et les banques de données s'intégrant à un réseau provincial de systèmes quasi autonomes reliés, au besoin, par liens de télécommunications à un centre serveur central.

\section{Réalisations et applications}

Dans le prolongement des systèmes de réservations exploités par les compagnies aériennes depuis plusieurs années, il faut également mentionner le développement, par Air Canada, d'un guichet automatique utilisant un lecteur de vidéodisque contrốlé par un micro-ordinateur relié à l'ordinateur central à Toronto pour superposer à l'image vidéo les informations sur les vols et permettre ainsi l'emission de billets sans l'intervention d'un agent.

Par ailleurs, le système Pegasus, que CP Air propose aux agences de voyages qui désirent s'équiper d'un ordinateur IBM-PC pour leur gestion interne et leurs réservations. mérite une mention particulière car l'approche bureautique adoptée nous sem= ble symptomatique d'une tendance qui laisse anticiper l'installation d'un assez grand nombre de terminaux susceptibles de permettre au besoin la consultation de $n$ 'importe quelle banque de données informatiscées.

Depuis cette époque, le Toronto Convention Center et quelques grands hotels se sont également équipés de systèmes d'affichage électronique dont l'envergure et les possibilites sont toutefois beaucoup plus limitées. Aux Etats-Unis, le New York Convention Center envisage présentement l'installation de lecteurs de vidéodisque contrôlés par micro-ordinateurs.

\section{Une demie-réussite}

Malheureusement, il faut aussi signaler l'abandon au printemps dernier du projet Téléguide, qui disposait de quelque 500 terminaux interactifs installés dans divers lieux publics à Toronto pour interroger une banque de données centrale contenant plus de 20000 pages d'information, mais qui, en dépit des efforts de ses promoteurs n'avait pas atteint le seuil d'une rentabilité commerciale satisfaisante après trois années d'exploitation. En Floride, le système Viewtron, lancé également en 1983, subissait lè même sort au début 1986 .

Faut-il conclure à l'échec définitif des réseaux vidéotex en Amérique du Nord?

\section{L'exemple européen}

Cela serait sans doute prématuré, car les Européens nous apportent l'exemple de deux projets d'envergure dont le succès est plus fermement établi

En Grande-Bretagne, le premier service vidéotex commercial au monde était lancé en 1979. Le service Prestel est disponible à tous les abonnés de la British Telecom, mais le reseau actuel comple plus de $85 \%$ $d^{\dagger} u t i l i s a t e u r s d^{\dagger}$ affaires sur un total de près de 200000 abonnés.

Parmi les différentes rubriques disponibles, la banque d'informations touristiques est certainement la plus populaire. On estime d'ailleurs à environ 6000 le nombre d'agences de voyages qui utilisent le réseau Prestel pour répondre aux demandes touchant les transports, les hôtels, les lieux de vacances, les messages, les réservations, les informations générales et les nouvelles. La création et la mise à jour des contenus sont effectuées par quelque 170 agents privés; ceux-ci sont les véritables fournisseurs des informations disponibles sur le réseau Prestel qui, de son côté, offre seulement la capacite de stockage et l'infrastructure des télécommunications.

Mais c'est l'expression d'une volonté politique qui a permis le lancement en France de Télétel, le service public de vidéotex interactif de grande envergure mis en place sous l'ćgide du Ministère des P.T.T. pour implanter tout d'abord l'annuaire électronique dans toutes les régions. Fin 1986 , plus de 2 millions et demi de Minitel, le terminal télématique de base des Français, seront en service sur le réseau Télétel. En conséquence, la gamme des services qui s'ajoutent à l'annuaire électronique s'est élargie avec la création de nouveaux ser-

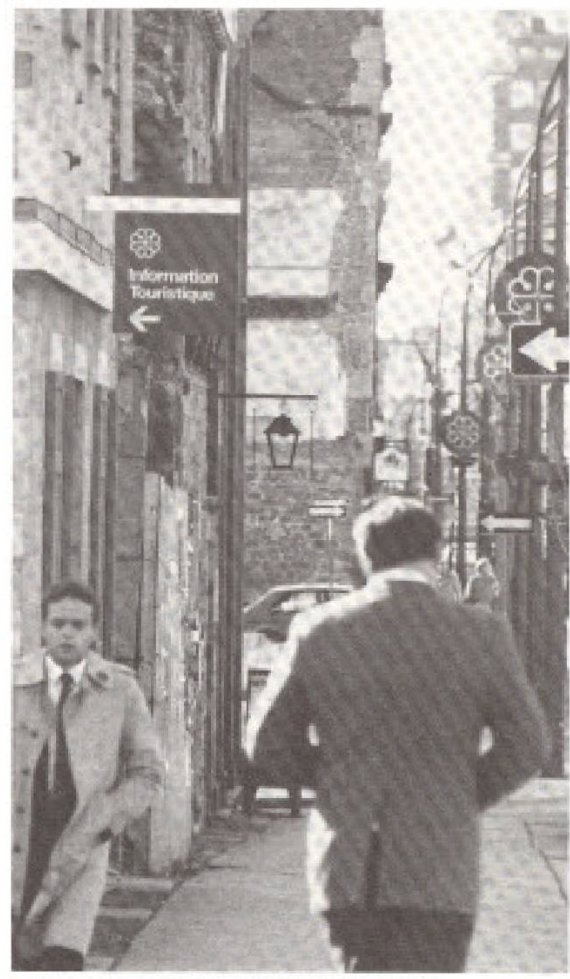

L'information touristique chez soi 
vices de messageries, de réservations et de consultation de banques de données qui répondent aux besoins des utilisateurs domestiques et professionnels. Il n'y a donc pas de doute, la télématique, au moins dans son activité vidéotex, a bien, en quatre ans, quitté la période expérimentale et fait son entrée comme instrument de communication dans la société française.

Au niveau des applications plus spécifiquement liées au tourisme, il est intéressant de souligner qu'après les services de messageries ce sont les services de réservations de billets d'avion et de chemin de fer qui sont parmi les plus populaires.

En outre, plusieurs municipalités françaises grandes et petites se mettent a I'heure télématique en attendant les services câblés. Il y avait, en 1985, près de 30 collectivités locales ou régionales qui proposaient à leurs administrés et aux touristes des informations sur le petit écran télématique.

Le contexte socio-économique est certes différent en France, et le Gouvernement français a joué, depuis 1978 , un rôle prépondérant en développant les infrastructures et en distribuant gratuitement les terminaux Minitel à la majorité des usagers. Le succès du projet démontre toutefois aujourd'hui la validité des concepts considérés hier encore comme avant-gardistes.

\section{La rentabilisation des investissements}

Au-delà de l'engouement technologique, le problème de la rentabilité commerciale des investissements publics et des coutt des services télématiques n'est pas encore véritablement résolu pour au moins deux raisons:

- les services et les banques de données ne sont pas assez nombreux ou diversifiés pour inciter les utilisateurs potentiels à acquérir le matériel nécessaire et à accepter toutes les conséquences financières de cet engagement;

- les services offerts ont été souvent créés par les innovations technologiques et les études de marché, de motivation et d'acceptabilité par les consommateurs sont souvent absentes lors des prises de décision sur le lancement des projets.

En effet, la concurrence règne et chacun veut faire comme son voisin pour garder son image de modernisme ou gagner sa place dans un marché mondial d'avenir.

Même en France, le marché apparaît encore peu préparé et toutes les applications ne se réalisent peut-être pas encore aujourd 'hui dans des conditions de rentabilité et de fiabilité indiscutables.

Au Canada, la plupart des tentatives de développement de systèmes vidéotex interactif donnant accès à des banques d'informations centralisées aussi bien que les projets de diffusion sur un canal de télévision ont connu l'échec ou un succès relatif, car le marché n'était pas prêt à assumer les coûts réels de ces services à la fin du programme de subventions du Ministère féderal des Communications.

\section{L'expérience que nous avons acquise au} Québec démontre d'ailleurs qu'une fois les problèmes techniques résolus, le véritable défi de la telématique n'est plus aujourd' hui dans la technologie elle-même mais plutốt dans tous les autres aspects humains, organisationnels et commerciaux qui conditionnent ultimement le succès ou l'échec de toute nouvelle entreprise.

\section{Les perspectives d'avenir}

Les applications potentielles de la télématique au tourisme sont étroitement liées au développement des systèmes d'information électronique. On peut en distinguer trois types principaux:

- les réseaux en circuit fermé, tel celui du Palais des Congrès de Montréal qui offre des possibilités intéressantes pour la diffusion d'une publicité centrée sur une clientèle homogène;

- les réseaux spécialisés destinés aux utilisateurs d'affaires, tels les systèmes de réservations et les systèmes de vente de billets de spectacles;

- les réseaux interactifs destinés au grand public, comme celui que Videoway se propose de développer à partir d'un projet pilote en cours d'expérimentation sur la rive sud de Montréal.

Quelques exemples américains démontrent que la publicité, dans les services de vidéotex, diffusée sur un canal de télévision peut apporter des revenus assez intéressants pour que leur promoteur ajoute des services et développe de nouveaux produits.

Par ailleurs, la diminution du coût des vidéodisques et des micro-ordinateurs permet d'entrevoir l'installation de systemes autonomes dans les hôtels et certains autres endroits fréquentés par les touristes.

Dans le contexte actuel et pour quelques années encore, nous ne pensons donc pas que les services télématiques auront pour l'industrie du tourisme au Québec une incidence sensible sur les marchés publicitaires traditionnels.

L'impact réel de ces technologies a moyen terme dépendra toutefois des formes de publicité nouvelles qui pourraient se développer, d'une part, avec la pénétration éventuelle de Videoway dans les réseaux de cabblodistribution et, d'autre part, avec le lancement toujours possible par Bell Canada d'un service vidéotex interactif de grande envergure utilisant le réseau téléphonique public de façon comparable à ce que les Français ont fait avec leur Minitel.
Qu'elle soit d'origine canadienne ou étrangère, la technologie est disponible et nous avons au Québec l'expérience requise pour la mettre en oeuvre dans les réseaux les plus sophistiqués. Mais force nous est d'admettre qu'il existe encore un grand décalage entre les possibilites techniques et l'usage qu'on en fait. Parmi les raisons qui expliquent cette situation, il faut souligner les points suivants:

- la résistance des hommes et des organisations aux changements et a l'innovation;

- la recherche, dans la technologie, de solutions qui relèvent plus souvent de l'organisation administrative;

- les difficultés de commercialisation de services sophistiqués qui restent méconnus de la plupart des intervenants et des professionnels;

- la publicité;

- le cloisonnement des disciplines et des entreprises dont la contribution est essentielle à la recherche de solutions globales.

\section{Le défi des années 1980}

Les difficultés qu'ont connues plusicurs projets vidéotex au Canada ne doivent pas ébranler notre foi dans les mérites du progrès. Elles doivent, au contraire, mobili= ser nos énergies et nos imaginations pour les surmonter et, ce faisant, elles posent aux dirigeants d'entreprises une question fondamentale: comment introduire dès aujourd'hui les nouvelles technologies de l'information dont leurs organisations. auront besoin demain pour répondre aux exigences de la clientèle ou pour simplement faire face à la concurrence?

Au-delà de l'aspect spectaculaire de certaines réalisations, il ne faut cependant pas perdre de vue les possibilités nouvelles que l'arrivée des ordinateurs personnels à bon marché et le développement des banques informatisées de renseignements accessibles à distance pourraient offrir à la majorité des petits intervenants du secteur touristique dont le rôle est si important dans la qualité et la personnalisation de l'accueil des touristes.

En guide de conclusion, nous soumettons à votre réflexion cette pensée de Machiavel écrite en 1513:

"Songez qu'il $n$ 'est d'affaire plus difficile, plus dangereuse à manier, plus incertaine de succes que d'entreprendre la creation de nouveaux systèmes.

Car le novateur a pour ennemis tous ceux que l'ordre ancien favorisait, et ne trouve que tiedes defenseurs chez ceux que favoriserait l'ordre nouveau. " 